\title{
Innovative Suspension System Integrated to the Wheel Rim for Lightweight Vehicle
}

\author{
Wojciech SKARKA ${ }^{1}$, Wojciech KOCOŃ, Ryszard SKOBERLA and Anna BZYMEK \\ Silesian University of Technology, Faculty of Mechanical Engineering, Poland
}

\begin{abstract}
Car industry has dynamically developed the electric car segment and is always on the lookout for new solutions that could help a company to become a leader brand on the market. Besides fully autonomous car technologies also new mechanical solutions could give advantage over other companies. Thus many ideas have been made to replace conventional vehicle suspension by the use of a flexible wheel rim. Based on over a year of experience, researches and studies in a field of existing lightweight vehicle suspension systems it was decided to design new solution, which structure eliminates main disadvantages of existing solutions such as energy losses and poor stability. The new suspension solution was designed based on our own knowledge and TRIZ method as a tool of verification. The CAD model of subassembly was subjected to a virtual examination for three different situations: standard ride, hard braking and sudden acceleration. Then a mathematical model of vibration damping properties has been created using MATLAB Simulink software. Additionally, the suspension quality and energy loss analysis have been performed to verify whether the problem was solved. The obtained results have been investigated and compared with commercially used car suspension systems.
\end{abstract}

Keywords. Suspension System, Simulation Model, TRIZ, TIPS, Innovative Solution

\section{Introduction}

The development of modern electric vehicles with electric drive sets new challenges. In electric vehicles, special attention is paid to energy consumption while driving, so as to ensure the best possible range of travel. Analyzing these problems, the aim is to improve the aerodynamics of the vehicle, the use of new more efficient propulsion systems and transmission of the drive as well as the energy stores [1]. Often the suspension of the vehicle goes down, which also has a major impact on energy consumption while driving. Not without significance is the fact that the suspension loads in electric vehicles are usually higher due to relatively large masses of energy storage. If one additionally impose the problem of providing steering and driving individual wheels, in vehicles with increased maneuverability, especially urban and autonomous, the vehicle suspension solutions used in the classical way barely meet the criteria for future-proof designs. Analyzing the requirements for future vehicles, the aim is to develop an innovative suspension solution that ensures the use of independent drive and steering of each individual wheel through a significant integration of the wheel suspension system allowing the suspension system to fit the wheel rim. Particular attention has been paid to the suspension design to minimize energy consumption while driving the vehicle

\footnotetext{
${ }^{1}$ Corresponding Author, Email: wojciech.skarka@polsl.pl.
} 
resulting from the work of the suspension itself. It is planned to use such a suspension in an ultra energy-efficient electric vehicle [2], [3].

\section{Innovative wheel suspension systems}

The assumed high degree of integration of the wheel suspension system drew the attention of the authors [4] to four innovative solutions used in light vehicles as well as off-road vehicles. These solutions (Fig. 1) are characterized by a common feature - they assume that the wheel rim is attached to the wheel hub by elastic elements of various types, i.e. bow spokes [5], loop spring elements [6], disk honeycomb structure [7] or eliptical spring elements [8] .

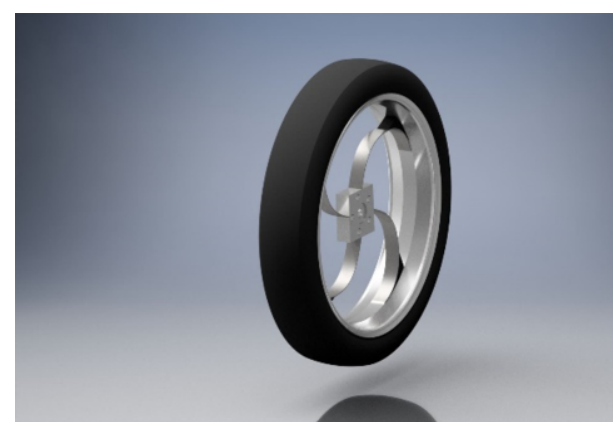

Chet Baigh concept [5]

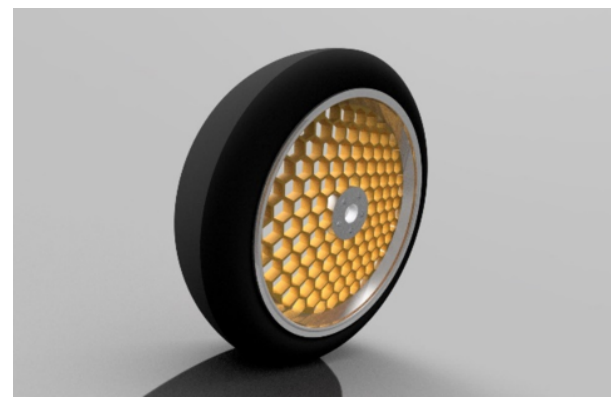

Honeycomb shock absorber wheel concept [7]

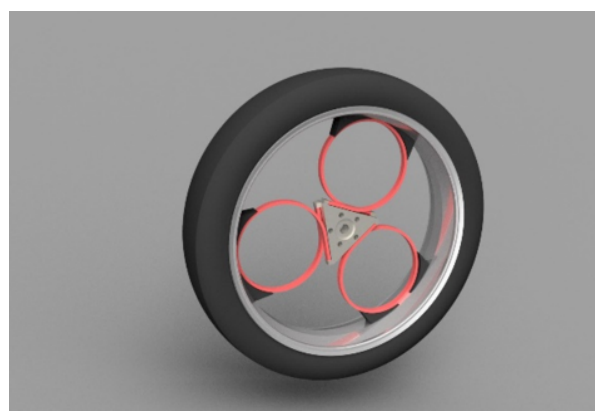

Loopwheel shock absorber concept [6]

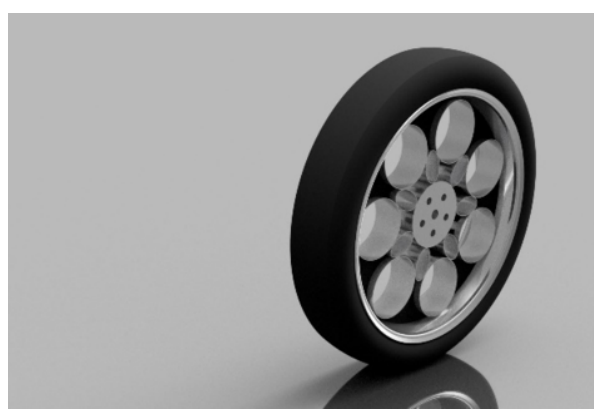

Elliptical spring wheel concept [8]

Figure 1. Various concepts of integrating suspension wheel system to the wheel disk.

In order to compare existing concepts of shock absorber lightweight structure wheels, several examination have been performed:

- $\quad$ Static structural analysis was performed to determinate the static stiffness of the whole system and behavior during normal driving conditions, hard braking (torsion load) and sudden acceleration. It was necessary for future calculations as the stiffness coefficient is one of the most influencing data related to suspension damping as well as torsional stiffness.

- Modal analysis was conducted to obtain the natural frequency of the system that is strongly related to vibration damping ability. 
- Mathematical model of suspension system allowed to compare the vibration damping properties of the systems.

In addition to determine suspension characteristics and simulate the work of suspensions of these types, the tests simulation models were also developed to calculate the energy consumption while driving with the use of such a suspension.

The research carried out by the authors to determine which solutions are the best suited for applications planned by the authors led to the conclusion that due to the large energy losses generated at each turn of the wheel, these solutions are not suitable [4] for use in an energy efficient electric vehicle [1], [2]. It is still possible to use these solutions in such application but they should be used in the different configuration.

\section{Identified problems of innovative suspension systems}

The basic problem of this kind of suspensions is the lack of integration possibilities in the system of springs connecting the rim and wheel hub to the damping elements. In this case, during each rotation of the wheel even in the absence of damped unevenness and rolling of the wheel on a completely flat terrain, the rotation of the elastically suspended axis and displacement of damping elements occurs during rotation.It results in expending additional energy for rotation of the wheel, what even more, in the absence of special elements suppressing the non-ideal characteristics of the suspension springs, causes similar phenomena, although in lower level, but during every wheel turn (Figure 2). Such inconveniences that cause energy consumption at each wheel turn do not allow this idea to be applied in this form. On the other hand, the necessity of a traditional way of assembling damping elements undermines the possibility of integrating the entire elastic dampening system into the space of the wheel disc. The whole benefit of this form of suspension is wasted.

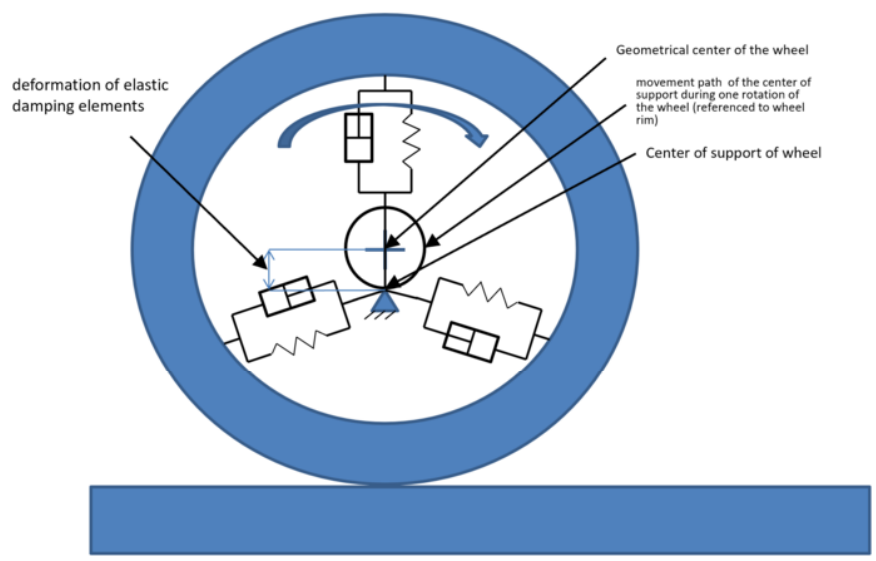

Figure 2. Identified problem of increased energy consumption

Another problem is the difficulty in guaranteeing the correct stering of the wheel. The proposed elastic suspension, shows considerable variability of compliance depending on the direction of the load. However, during the operational loading of the disc consisting of flexible elements in directions other than the plane of the target unfavorable deformation causes difficulties in steering the wheel. In addition, during the 
loading of the twisting moment that results from braking or driving the wheel, the disc is twisted and additional reversal or torsional vibrations occur. Such a suspension tends to have unfavorable self-excited vibrations in many directions.

TRIZ/TIPS (Theory of Inventive Problem Solving) ideation method [9] was used to find a solution to the problem described.. Thus, a solution was created for an innovative method of eliminating the main drawback of the above mentioned solutions with the simultaneous use of their basic advantages.

\section{Concept of innovative suspension system}

Considering the identified problems it was decided to develop another innovative solution, which keeps as much as possible of advantages of existing solutions, but does not have its disadvantages. The basic issue that had to be solved was to eliminate the need of spinning the elastic-damping disc. This solution would allow to avoid an adverse effect of consantly changing disc deformation. As an assumption in design process, the compactness of the developed solution and the integration of components included in the suspension system were also adopted.

It was necessary to develop a solution, that would have all advantages of the elasticdamping disc, especially putting it in inner space of the rim, but without possibility of spinning with the rim. During the conceptual phase it was decided to use one of the most effecive ideation methods, which is the TRIZ method. It turned out that in the case of the developed solution, the method of inversion is very useful. Precisely, this is a case of immobilizing the rotating element and setting the immobilized element in motion. Until now, on the immobilized axis there was a rotating elastic-damping disc that served as a connection between the rim and the wheel hub. The ring inside the shield was immobilized, while the outer part moved with respect to the inner ring together with the rim. In the described solution, it was proposed to immobilize the outer ring and to give the inner ring the possibility to rotate with half shaft embedded in it. The discussed solution is presented in Fig. 3.

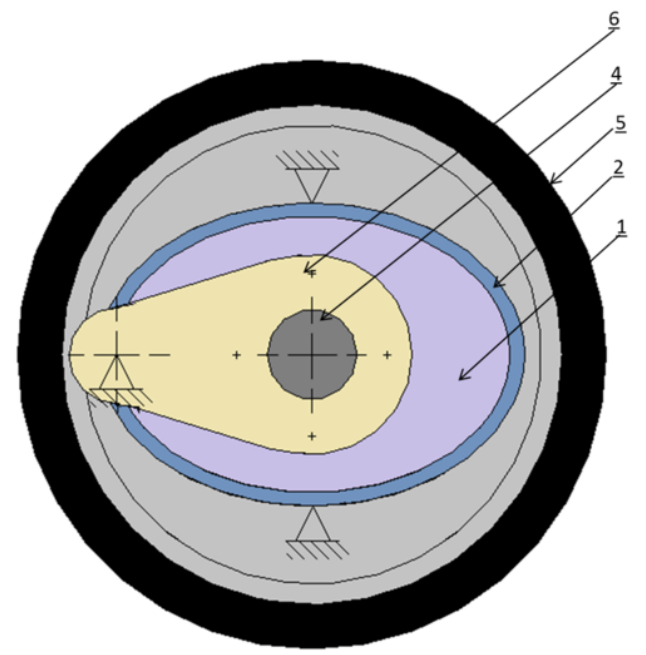

Figure 3. The concept of suspension system 
In the proposed solution [10] the elastic-damping disc 2 is not a part of the wheel rim 5, but is a separate element. The wheel axle is mounted in the inner ring of the disc. The outer ring of the disc is attached to the adapter. The swingarm 6 is also attached to the adapter. The adapter allows to mountto the wheel to the vehicle frame. Due to this solution, the suspension system becomes very compact. In addition, this system is an closed system. In the state that was presented in Fig. 3, it is an functional system, without the necessity of fixing as in the case of traditional suspension systems. The suspension system described above can be used for both non-propelled and propelled wheels.
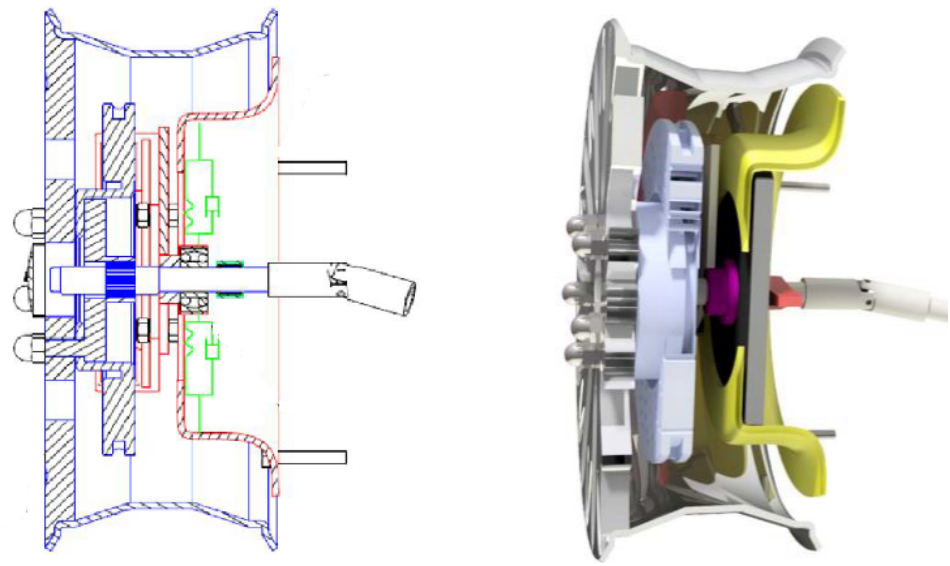

Figure 4a. Detailed concept of suspension system b. The model of suspension system with composite disc.

Figure 4a presents a detailed illustration of the suspension concept. The suspension system consists of a disc with elastic-damping characteristics and flexibility in one direction. The disk is attached to the ring, which is the element that connects the system to the vehicle frame. A bearing unit through which the wheel axle passes is built into the hole of the elastic-damping disc. The wheel axle is mounted in the rocker arm connected to the elements of the ring. Due to this the wheel has only two degrees of freedom, rotation around its own axis and vertical motion.

Figure $4 \mathrm{~b}$, Figure $5 \mathrm{a}$ and Figure $5 \mathrm{~b}$ present the models of physical realizations of the described concept but with different solutions for elastic-damping disc. In Fig. 5, the elastic-damping element is a full composite disk with appropriate material properties. Figure 5a shows a model in which the elastic-damping element is the honeycomb structure similar to the solution presented erlier [7] but improved for the purpose of energy efficient application [10], [2].

In Figure 5b, the elastic-damping element is a disk consisting of rolled-up elastic strips connected to the hub in a manner that allows shock absorbing and damping. The solution is similar to presented in the loopweel design [6] but significantly improved [10]. The analyzed solutions are perfectly suited to the development of the Generative Modeling methods used so far in the design of vehicles [11].

\section{Numerical simulations and its results}

In order to compare the properties of innovative suspension solutions, virtual models of suspension were made and numerical simulations were performed for analyses such as: static structural analysis, modal analysis. Afterwards mathematical model of the 
suspension was developed, and tests of the damping properties of selected solutions were performed. Structures chosen for analysis were: composite disc, Loopwheel, Honeycomb [12]. Below the approach to three types of analyses with its assumptions were presented and the results were discussed.
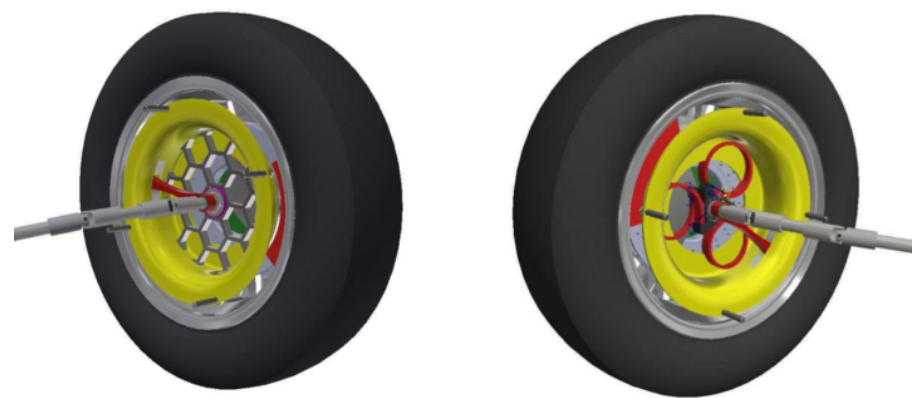

Figure 5. The model of suspension system with a) honeycomb structure disc, b) special loopwheel disc.

\section{Static structural analysis}

The static structural analysis was performed INVENTOR program that allowed to determine the stresses, displacements and deformations of the entire suspension system caused by the applied load for given boundary conditions. The simulation was performed for three different situations, such as: normal driving conditions, hard braking and sudden acceleration. The conditions were chosen in such a way as to predict the behavior of the system during constant driving and in extreme situations that cause temporary overload of the system. Static structural analysis provides important data on the highest stresses and displacement values. Thanks to this, if the values of operating forces are known, it is possible to calculate the stiffness and basing on this, toassume the stability of the system, which is necessary for further calculations.

In vehicle suspension systems, the basic static stiffness of the wheel in the normal direction is essential because it determines the stability and functionality of the suspension system. The results of the structural analysis showed that the models with the Loopwheel system and the Honeycomb structure behave slightly differently in the conditions of normal driving on a flat road and in the case of sudden acceleration and hard braking than the composite disc. Both models showed lower values of displacements in each of the three examined cases, which proves their greater stiffness. The results of this study showed that the newly designed concept is capable of carrying large loads during hard braking and will not lose its stability.

Table 1. The comparision of results - Stress [MPa].

\begin{tabular}{lccc}
\hline Driving style & Composite disc & Loopwheel & Honeycomb \\
\hline Normal driving & 57,44 & 66,77 & 32,57 \\
\hline Hard braking & 111,45 & 164,21 & 79,65 \\
\hline Sudden acceleration & 92,35 & 114,21 & 54,33 \\
\hline
\end{tabular}


Table 2. The comparision of results - Displacement [mm].

\begin{tabular}{lccc}
\hline Driving style & Composite disc & Loopwheel & Honeycomb \\
\hline Normal driving & 15,41 & 11,94 & 9,35 \\
\hline Hard braking & 21,00 & 14,32 & 9,23 \\
\hline Sudden acceleration & 17,13 & 11,65 & 7,22 \\
\hline
\end{tabular}

As it can be seen from the results presented in Tables 1 and 2, composite disk and a Loopwheel models are not as rigid as the honeycomb model, which has significantly lower displacement values. Another important feature is the behavior of the wheel and suspension system during rapid acceleration and hard braking. From the results presented in Tables 1 and 2, it can be seen that the load conditions in the wheels is much larger than in the case of normal driving on a flat road. The above analysis shows that the model with honeycomb structure [12] shows the lowest displacements and stresses in the state of a complex load.

\section{Numerical modal analysis}

The next performed study was a modal analysis that allowed even more accurate determination of the object's stability. Numerical modal analysis, as a study of the dynamic properties of the entire object, allowed to determine the natural frequency of the object vibrations. The boundary conditions simulated the release of all degrees of freedom.

The results of numerical modal analysis included six modes of natural frequencies, of which the first (the lowest) values were used as inputs for a mathematical model, and were presented in the Table 3 . The results indicated that the newly designed concepts have higher natural frequencies than the composite disc model.

Table 3. The comparision of results - Natural frequency $[\mathrm{Hz}]$.

\begin{tabular}{lccc}
\hline & Composite disc & Loopwheel & Honeycomb \\
\hline Natural frequency $[\mathrm{Hz}]$ & 19,03 & 34,93 & 23,50 \\
\hline
\end{tabular}

The lowest natural frequency occurs subsequentially for the composite disc, Honeycomb and Loopwheel. These values have an effect on the stability of the system and driving comfort, however, all values obtained are correct (in the range from 0 to $80 \mathrm{~Hz}$ ) for the suspension system in an accordance with regulations in ISO 2631-1: 1997.

\section{Mathematical model and numerical simulation model of the suspension system}

The mathematical model of a system is a fundamental tool that provides a lot of information about the main features of the internal suspension system and the entire wheel. In the case of collecting data about the damping properties of the newly designed flexible composite wheel disk, it was necessary to calculate the stiffness of the wheel. This task was carried out using PATRAN software. The results obtained from previous simulations (structural analysis and modal analysis) were used as an input to the mathematical model of the suspension system. To properly calculate the vibration 
damping properties of the wheel, the equations for the harmonic oscillator were used. In fig. 6 the numerical model of suspension system was presented. This module of the simulation model is the basis for completing the simulation model of the whole vehicle [13].

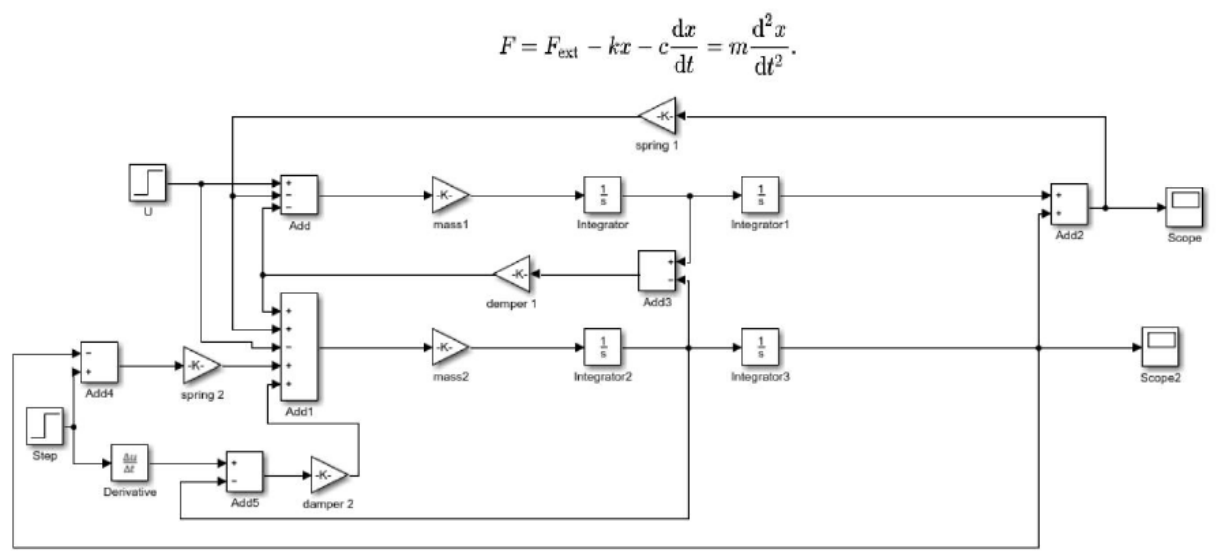

Figure 6. Mathematical model and numerical model of the suspension system.

\section{Results of damping properties verification}

The simulations with use of the numerical model of the suspension system, allowed to determine the damping properties of the analysed wheel models. The expected value of the damping factor strongly depends on the vehicle application. The graph shown (Fig. 7) shows the behavior of a lightweight vehicle $(600 \mathrm{Kg}+$ mass of hybrid wheels) during ride on a flat road. As can be observed, the composite disc and the Loopwheel have very similar damping properties, vibrations are much higher in frequency than in the case of Honeycomb system, the oscillation has a higher amplitude but the system returns to equilibrium faster. In the case of Honeycomb, the oscillation is less violent and is characterized by a lower frequency. The system quickly and smoothly returns to balance, which provides a more pleasant and comfortable ride.

The described behavior was confirmed also by the value of the calculated damping ratio ( presented in Table 4), which is close to the expected value of 1 for the Honeycomb structure.

The summary of the performed tests using virtual prototyping methods was optimization aimed at finding a system that best meets all the requirements set before it, represented by four fundamental criteria: damping, steering, sudden braking and acceleration, total weight. 


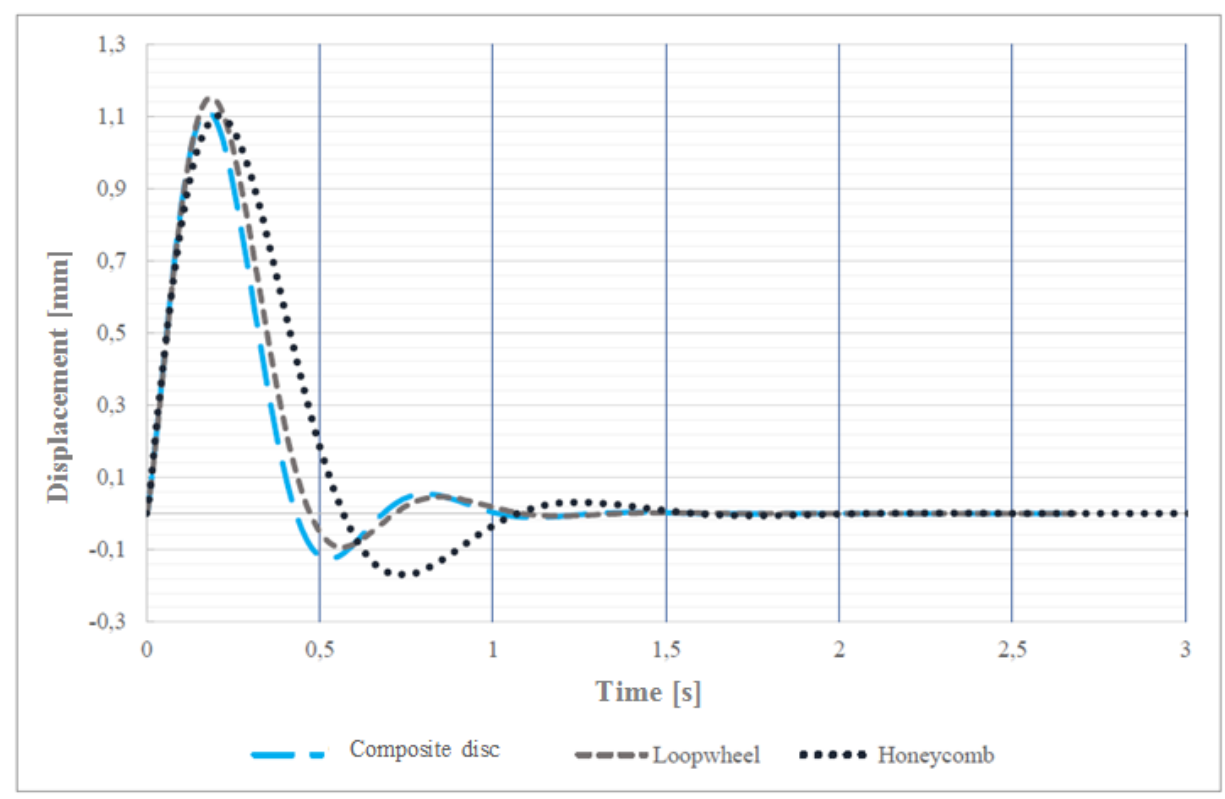

Figure 7. A graph of vibration damping properties for each model.

Table 4. The comparision of results - damping ratio.

\begin{tabular}{lccc}
\hline & Composite disc & Loopwheel & Honeycomb \\
\hline Damping ratio & 0,52 & 0,57 & 0,77 \\
\hline
\end{tabular}

\section{Conclusions}

Electric vehicles, especially those with high maneuverability, pose new challenges for suspension systems. The authors have developed a new compact suspension system integrated into the wheel space combining the most important advantages of the previously proposed systems and eliminating their serious drawbacks. The tests showed the correctness of the proposed solutions and the structure of Honeycomb was selected as the best structure for the elastic-damping disc. In further works it is planned to optimize this solution and to integrate electroactive elements to increase the suspension potential by introducing the possibility of an active change in the characteristics and reacting to the road conditions encountered. This active suspension integrated into the wheel space is the ideal solution for newly designed electric and autonomous vehicles. The inventive solution proposed in the article has been submitted for patent protection. In the new suspension solution, the suspension disc element with elastic-damping characteristics becomes critical. Various shapes of this suspension disc were considered, but no detailed aspects of materials used on this target have been analyzed so far. As it is supposed to have damping properties, further analysis and selection of appropriate materials guaranteeing such characteristics is required. Therefore, material tests or the design of an appropriate form and combination of shield materials are required. Further research will be carried out in this direction. 


\section{Acknowledgement}

Publication partially supported as a part of the Rector's grant in the area of scientific research and development works. Silesian University of Technology, grant number 10/060/RGJ18/0082

\section{References}

[1] W. Skarka, Reducing the Energy Consumption of Electric Vehicles, Advances in Transdisciplinary Engineering, Vol. 2, IOS Press, Amsterdam, 2015, pp. 500-509.

[2] T.Pabian, W.Skarka, An optimization approach for an ultra-efficient electric racing vehicle's supporting system based on composite shell elements. EngOpt 2018. Proceedings of the 6th International Conference on Engineering Optimization, September 17-19, 2018, Lisbon, Portugal. Eds.: H. C. Rodrigues, J. Herskovits, C. M. Mota Soares, A. L. Araujo, J. M. Guedes, J. O. Folgado, F. Moleiro, J. F. A. Madeira. Springer, Cham, 2019, pp. 1061-1072.

[3] W. Skarka, Methodology for the optimization of an energy efficient electric vehicle, Proceedings of the 6th International Conference on Integrity-Reliability-Failure. IRF2018, Lisbon/Portugal, 22-26 July 2018. Eds.: J. F. Silva Gomes, Shaker A. Meguid. [Porto] : FEUP-INEGI, 2018, pp. 415-422

[4] W. Kocoń and W. Skarka, Hybrid wheel innovative suspension system. Advances in Transdisciplinary Engineering, Vol. 7, IOS Press, Amsterdam, 2018, pp. 496-505.

[5] T. Berman, ShockWheel: A Revolutionary Wheel Technology With Bow-Spokes, Interesting Engineering, 15 ${ }^{\text {th }}$ January 2017, https://interestingengineering.com/shockwheel-a-revolutionary-wheeltechnology-with-bow-spokes, Accessed July 8, 2019.

[6] Loopwheel, 2018, Loopwheel.com, http://www.goesbike.com/articles/2400/1/Loopwheel-ban-sepedadengan-suspensi-sendiri.html, Accessed: Jan 19, 2018.

[7] Q. Zhang, X. Yang, P. Li, G. Huang, S. Feng, Ch. Shen, Bioinspired engineering of honeycomb structure - Using nature to inspire human innovation, Progress in Materials Science, 2015, Vol. 74, pp. 332-400.

[8] D. Cardile, N Viola, S. Chiesa and A. Rougier, Applied design methodology for lunar rover elastic wheel, Acta Astronautica, 2012, Vol. 81, pp. 1-11.

[9] TRIZ https://en.wikipedia.org/wiki/TRIZ

[10] W. Skarka, R.Skoberla: Vehicle wheel suspension system - Application for patent P. 429743 pending

[11] A.Jałowiecki, W. Skarka, Generative modeling in ultra-efficient vehicle design. Advances in Transdisciplinary Engineering, Vol. 4, IOS Press, Amsterdam, 2016, pp. 999-1008.

[12] M.John, A.John, W.Skarka, The influence of the geometrical parameters on the mechanical properties of honeycomb structure. MECHANIKA, 2017, Vol. 23, Issue: 3, pp. 359-364.

[13] M. Targosz, W. Skarka and P. Przystałka, Model-Based Optimization of Velocity Strategy for Lightweight Electric Racing Cars, Journal of Advanced Transportation, Volume 2018, Article ID 3614025, 20 pages https://doi.org/10.1155/2018/3614025 